\title{
Physically Inspired Models for the Synthesis of Stiff Strings with Dispersive Waveguides
}

\author{
I. Testa \\ Dipartimento di Scienze Fisiche, Università di Napoli “Federico II,” Complesso Universitario di Monte S. Angelo, 80126 Napoli, Italy \\ Email: italo.testa@na.infn.it \\ G. Evangelista \\ Dipartimento di Scienze Fisiche, Università di Napoli “Federico II," Complesso Universitario di Monte S. Angelo, 80126 Napoli, Italy \\ Email: gianpaolo.evangelista@na.infn.it

\section{S. Cavaliere} \\ Dipartimento di Scienze Fisiche, Università di Napoli “Federico II," Complesso Universitario di Monte S. Angelo, 80126 Napoli, Italy \\ Email: cavaliere@na.infn.it
}

\author{
Received 30 June 2003; Revised 17 November 2003
}

\begin{abstract}
We review the derivation and design of digital waveguides from physical models of stiff systems, useful for the synthesis of sounds from strings, rods, and similar objects. A transform method approach is proposed to solve the classic fourth-order equations of stiff systems in order to reduce it to two second-order equations. By introducing scattering boundary matrices, the eigenfrequencies are determined and their $n^{2}$ dependency is discussed for the clamped, hinged, and intermediate cases. On the basis of the frequency-domain physical model, the numerical discretization is carried out, showing how the insertion of an all-pass delay line generalizes the Karplus-Strong algorithm for the synthesis of ideally flexible vibrating strings. Knowing the physical parameters, the synthesis can proceed using the generalized structure. Another point of view is offered by Laguerre expansions and frequency warping, which are introduced in order to show that a stiff system can be treated as a nonstiff one, provided that the solutions are warped. A method to compute the all-pass chain coefficients and the optimum warping curves from sound samples is discussed. Once the optimum warping characteristic is found, the length of the dispersive delay line to be employed in the simulation is simply determined from the requirement of matching the desired fundamental frequency. The regularization of the dispersion curves by means of optimum unwarping is experimentally evaluated.
\end{abstract}

Keywords and phrases: physical models, dispersive waveguides, frequency warping.

\section{INTRODUCTION}

Interest in digital audio synthesis techniques has been reinforced by the possibility of transmitting signals to a wider audience within the structured audio paradigm, in which algorithms and restricted sets of data are exchanged [1]. Among these techniques, the physically inspired models play a privileged role since the data are directly related to physical quantities and can be easily and intuitively manipulated in order to obtain realistic sounds in a flexible framework. Applications are, amongst the others, the simulation of a "physical situation" producing a class of sounds as, for example, a closing door, a car crash, the hiss made by a crawling creature, the human-computer interaction and, of course, the simulation of musical instruments.

In the general physical models technique, continuoustime solutions of the equations describing the physical sys- tem are sought. However, due to the complexity of the real physical systems-from the classic design of musical instruments to the molecular structure of extended objectssolutions of these equations cannot be generally found in an analytic way and one should resort to numerical methods or approximations. In many cases, the resulting approximation scheme only closely resembles the exact model. For this reason, one could better define these methods as physically inspired models, as first proposed in [2], where the mathematical equations or solutions of the physical problem serve as a solid base to inspire the actual synthesis scheme. One of the advantages of using physically inspired models for sound synthesis is that they allow us to perform a "selection" of the physical parameters actually influencing the sound so that a trade-off between completeness and particular goals can be achieved. 
In the following, we will focus on stiff vibrating systems, including rods and stiff strings as encountered in pianos. However, extensions to two- or three-dimensional systems can be carried out with little effort.

Vibrating physical systems have been extensively studied over the last thirty years for their key role in many musical instruments. The wave equation can be directly approximated by means of finite difference equations $[3,4,5,6,7]$, or by discretization of the wave functions as proposed by Jaffe and Smith $[8,9]$ who reinterpreted and generalized the Karplus-Strong algorithm [10] in a wave propagation setting. The outcome of the approximation of the time domain solution of the wave equation is the design of a digital waveguide simulating the string itself: the sound signal simulation is achieved by means of an appropriate excitation signal, such as white noise. However, in order to achieve a more realistic and flexible synthesis, the interaction of the excitation system with the vibrating element is, in turn, physically modeled. Digital waveguide methods for the simulation of physical models have been widely used $[11,12,13,14,15,16]$. One of the reasons for their success is that they are appropriate for real-time synthesis $[17,18,19,20]$. This result allowed us to change our approach to model musical instruments based on vibrating strings: waveguides can be designed for modeling the "core" of the instruments, that is, the vibrating string, but they are also suitable for the integration of interaction models, for example, for the excitation due to a hammer [21] or to a bow [9], the radiation of sound due to the body of the instrument $[22,23,24,25]$, and of different side-effects in plucked strings [26]. It must be pointed out that the interactions being highly nonlinear, their modeling and the determination of the range of stability is not an easy task.

In this paper, we will review the design of a digital waveguide simulating a vibrating stiff system, focusing on stiff strings and treating bars as a limit case where the tension in negligible. The purpose is to derive a general framework inspiring the determination of a discrete numerical model. A frequency domain approach has been privileged, which allows us to separate the fourth-order differential equation of stiff systems into two second-order equations, as shown in Section 2. This approach is also useful for the simulation of two-dimensional (2D) systems such as thin plates. By enforcing proper boundary conditions, we obtain the eigenfrequencies and the eigenfunctions of the system as found, for the case of strings, in the classic works by Fletcher $[27,28]$. Once the exact solutions are completely characterized, their numerical approximation is discussed $[29,30]$ together with their justification based on physical reasoning. The discretization of the continuous-time domain solutions is carried out in Section 3, which naturally leads to dispersive waveguides based on a long chain of all-pass filters. From a different point of view, the derived structure can be described in terms of Laguerre expansions and frequency warping [31]. In this framework, a stiff system can be shown to be equivalent to a nonstiff (Karplus-Strong like) system, whose solutions are frequency warped, provided that the initial and the possibly moving boundary conditions are properly unwarped $[32,33]$. As a side effect, this property can be exploited in order to perform an analysis of piano sounds by means of pitch-synchronous frequency warped wavelets in which the excitation can be separated from the resonant sound components [34].

The models presented in this paper provide at least two entry points for the synthesis. If the physical parameters and boundary conditions are completely known, or if it is desired to specify them to model arbitrary strings or rods, then the eigenfunctions, hence the dispersion curve, can be determined. The problem is then reconducted to that of finding the best approximation of the continuous-time dispersion curve with the phase response of a suitable all-pass chain using the methods illustrated in Section 3. Another entry point is offered if sound samples of an instrument are available. In this case, the parameters of the synthesis model can be determined by finding the warping curve that best fits the data given by the frequencies of the partials, together with the length of the dispersive delay line. This is achieved by means of a regularization method of the experimental dispersion data, as reported in Section 4.

The physical entry point is to be preferred in those situations where sound samples are not available, for example, when we are modeling a nonexisting instrument by extension of the physical model, such as a piano with unusual speaking length. The other entry level is best for approximating real instrument sounds. However, in this case, the synthesis is limited to existing sources, although some variations can be obtained in terms of the warping parameters, which are related to, but do not directly represent, physical factors.

\section{PHYSICAL STIFF SYSTEMS}

In this section, we present a brief overview of the stiff string and rod equations of motion and of their solution. The purpose is twofold. On the one hand, these equations give the necessary background to the physical modeling of stiff strings. On the other hand, we show that their frequency domain solution ultimately provides the link between continuous-time and discrete-time models, useful for the derivation of the digital waveguide and suitable for their simulation. This link naturally leads to Laguerre expansions for the solution and to frequency warping equivalences. Furthermore, enforcing proper boundary conditions determines the eigenfrequencies and eigenfunctions of the system, useful for fitting experimentally measured resonant modes to the ones obtained by simulation. This fit allows us to determine the parameters of the waveguide through optimization.

\subsection{Stiff string and bar equation}

The equation of motion for the stiff string can be determined by studying the equilibrium of a thin plate $[35,36]$. One obtains the following 4 th-order differential equation for the deformation of the string $y(x, t)$ :

$$
\begin{aligned}
-\varepsilon \frac{\partial^{4} y(x, t)}{\partial x^{4}}+\frac{\partial^{2} y(x, t)}{\partial x^{2}} & =\frac{1}{c^{2}} \frac{\partial^{2} y(x, t)}{\partial t^{2}}, \\
\varepsilon=\frac{E I}{T}, \quad c & =\sqrt{\frac{T}{\rho S}},
\end{aligned}
$$


featuring the Young modulus of the material $E$, the inertia moment $I$ with respect to the transversal axis of the crosssection of the string (for a circular section of radius $r, I=$ $\pi r^{4} / 4$ as in [36]), the tension of the string $T$, and the mass per unit length $\rho S$. Note that for $\varepsilon \rightarrow 0$, (1) becomes the wellknown equation of the vibrating string [35]. Otherwise, if the applied tension $T$ is negligible, we obtain

$$
-\varepsilon^{\prime} \frac{\partial^{4} y(x, t)}{\partial x^{4}}=\frac{\partial^{2} y(x, t)}{\partial t^{2}}, \quad \varepsilon^{\prime}=\frac{E I}{\rho S},
$$

which is the equation for the transversal vibrations of rods. Solutions of (1) and (2) are best found in terms of the Fourier transform of $y(x, t)$ with respect to time:

$$
Y(x, \omega)=\int_{-\infty}^{+\infty} y(x, t) \exp (-i \omega t) d t,
$$

where $\omega$ is the angular velocity related to frequency $f$ by the relationship $f=2 \pi \omega$.

By taking the Fourier transform of both members of (1) and (2), we obtain

$$
\varepsilon \frac{\partial^{4} Y(x, \omega)}{\partial x^{4}}-\frac{\partial^{2} Y(x, \omega)}{\partial x^{2}}-\frac{\omega^{2}}{c^{2}} Y(x, \omega)=0
$$

for the stiff string and

$$
\varepsilon^{\prime} \frac{\partial^{4} Y(x, \omega)}{\partial x^{4}}-\omega^{2} Y(x, \omega)=0
$$

for the rod.

The second-order $-\partial^{2} / \partial x^{2}$ spatial differential operator is defined as a repeated application of the $L_{2}$ space extension of the $-i(\partial / \partial x)$ operator [37]. To the purpose, we seek solutions whose spatial and frequency dependency can be factored, according to the separation of variables method, as follows:

$$
Y(x, \omega)=W(\omega) X(x) .
$$

Substituting (6) in (4) and (5) results in the elimination of the $W(\omega)$ term, obtaining ordinary differential equations, whose characteristic equations, respectively, are

$$
\begin{aligned}
\varepsilon \lambda^{4}-\lambda^{2}-\frac{\omega^{2}}{c^{2}} & =0 & & \text { (stiff string) } \\
\varepsilon^{\prime} \lambda^{4}-\omega^{2} & =0 & & \text { (rod) }
\end{aligned}
$$

The elementary solutions for the spatial part $X(x)$ have the form $X(x)=C \exp (\lambda x)$. It is important to note that in both cases, the characteristics equations have the following form:

$$
\left(\lambda^{2}-\xi_{1}^{2}\right)\left(\lambda^{2}-\xi_{2}^{2}\right)=0,
$$

where $\xi_{1}$ and $\xi_{2}$ are, in general, complex numbers that depend on $\omega$. Equation (8) allows us to factor both equations in (4) and (5) as follows:

$$
\left[\frac{\partial^{2}}{\partial x^{2}}-\xi_{1}^{2}\right] \cdot\left[\frac{\partial^{2}}{\partial x^{2}}-\xi_{2}^{2}\right] Y(x, \omega)=0 .
$$

The operator $-\partial^{2} / \partial x^{2}$ is selfadjoint with respect to the $L_{2}$ scalar product [37]. Therefore, (9) can be separated into the following two independent equations:

$$
\begin{aligned}
& {\left[\frac{\partial^{2}}{\partial x^{2}}-\xi_{1}^{2}\right] Y_{1}(x, \omega)=0,} \\
& {\left[\frac{\partial^{2}}{\partial x^{2}}-\xi_{2}^{2}\right] Y_{2}(x, \omega)=0,}
\end{aligned}
$$

where

$$
Y(x, \omega)=Y_{1}(x, \omega)+Y_{2}(x, \omega) .
$$

As we will see, (10) justifies the use, with proper modifications, of a second-order generalized waveguide based on progressive and regressive waves for the numerical simulation of stiff systems.

\subsection{General solution of the stiff string and bar equations}

In this section, we will provide the general solution of (8). The particular eigenfunctions and eigenfrequencies of rods and stiff strings are determined by proper boundary conditions and are treated in Section 2.3. From (7), it can be shown that

$$
\begin{aligned}
& \xi_{1}^{ \pm}= \pm \sqrt{-\frac{\sqrt{1+4 \omega^{2} \varepsilon / c^{2}}-1}{2 \varepsilon}} \quad \text { (stiff string), } \\
& \xi_{2}^{ \pm}= \pm \sqrt{\frac{\sqrt{1+4 \omega^{2} \varepsilon / c^{2}}+1}{2 \varepsilon}} \quad \text { } \\
& \xi_{1}^{ \pm}= \pm \sqrt{-\frac{\omega}{\sqrt{\varepsilon^{\prime}}}} \quad(\operatorname{rod}) . \\
& \xi_{2}^{ \pm}= \pm \sqrt{\frac{\omega}{\sqrt{\varepsilon^{\prime}}}} \quad \text {. }
\end{aligned}
$$

Note that in both cases, the eigenvalues $\xi_{1}^{ \pm}$are complex numbers, while $\xi_{2}^{ \pm}$are real numbers. It is also worth noting that

$$
\begin{array}{ll}
\xi_{1}^{2}+\xi_{2}^{2}=\frac{1}{\varepsilon} & (\text { stiff string }) \\
\xi_{1}^{2}+\xi_{2}^{2}=0 & (\operatorname{rod}),
\end{array}
$$

where $\xi_{1}$ corresponds to the positive choice of the sign in front of the square root in (12) and $\xi_{2}=\left|\xi_{2}^{ \pm}\right|$. As expected, if we let $T \rightarrow 0$, then both sets of eigenvalues of the stiff string tend to those found for the rod. Using the equations in (12), we then have for both strings and rods

$$
\begin{aligned}
& Y_{1}(x, \omega)=c_{1}^{+} \exp \left(\xi_{1} x\right)+c_{1}^{-} \exp \left(-\xi_{1} x\right) \\
& Y_{2}(x, \omega)=c_{2}^{+} \exp \left(\xi_{2} x\right)+c_{2}^{-} \exp \left(-\xi_{2} x\right)
\end{aligned}
$$

where $c_{1}^{ \pm}, c_{2}^{ \pm}$are, in general, functions of $\omega$. Note that $Y_{1}(x, \omega)$ is an oscillating term, while, since $\xi_{2}$ is real, $Y_{2}(x, \omega)$ is nonoscillating. For finite-length strings, both positive and negative real exponentials are to be retained. 
From (12), we see that the primary effect of stiffness is the dependency on frequency of the argument (from now on, phase) of the solutions of (4) and (5). Therefore, the propagation of the wave from one section of the string located at $x$ to the adjacent section located at $x+\Delta x$ is obtained by multiplication of a frequency dependent factor $\exp \left(\xi_{1} \Delta x\right)$. Consequently, the group velocity $u$, defined as $u \equiv\left(d \xi_{1} / d \omega\right)^{-1}$, also depends on frequency. This results in a dispersion of the wave packet, characterized by the function $\xi_{1}(\omega)$, whose modulus is plotted in Figure 1 for the case of a brass string using the following values of the physical parameters $r, T, \rho$, and $E$ :

$$
\begin{aligned}
r & =1 \mathrm{~mm}, \\
T & =9 \cdot 10^{7} \text { dyne, } \\
\rho & =8.44 \mathrm{~g} \mathrm{~cm}^{-3}, \\
E & =9 \cdot 10^{11} \text { dyne } \mathrm{cm}^{-2} .
\end{aligned}
$$

Clearly, the previous example is a very crude approximation of a physical piano string (e.g., real-life piano strings in the low register are built out of more than one material and a copper or brass wire is wrapped around a steel core). For the sake of completeness, we give the explicit expression of $|u|$ in both the cases we are studying. We have

$$
\begin{aligned}
& |u|=\frac{2 c \sqrt{\left(c^{2}+4 \omega^{2} \varepsilon\right)}}{\sqrt{\left(2 c^{2} \pm 2 c \sqrt{\left(c^{2}+4 \omega^{2} \varepsilon\right)}\right)}} \quad \text { (stiff string), } \\
& |u|=2 \sqrt{\omega \sqrt{\varepsilon^{\prime}}} \quad(\operatorname{rod}) .
\end{aligned}
$$

If $T \rightarrow 0$, the two group velocities are equal. Moreover, if in the first line in (16), we let $\varepsilon \rightarrow 0$, then $u \rightarrow c$, which is the limit case of the ideally flexible vibrating string. These facts further justify the use of a dispersive waveguide in the numerical simulation. With respect to this point, a remark is in order: the dispersion introduced by stiffness can be treated as a limiting "nonphysical" consequence of the Euler-Bernoulli beam equation:

$$
\frac{d^{2}}{d x^{2}}\left[E I \frac{d^{2} y}{d x^{2}}\right]=p
$$

where $p$ is the distributed load acting on the beam. It is "nonphysical" in the sense that $u \rightarrow \infty$ as $\sqrt{\omega}$. However, in the discrete-time domain, this "nonphysical" situation is avoided if we suppose all the signals be bandlimited.

\subsection{Complete characterization of stiff string and rod solution}

Boundary conditions for real piano strings lie in between the conditions of clamped extrema:

$$
\begin{aligned}
Y\left(-\frac{L}{2}, \omega\right) & =Y\left(\frac{L}{2}, \omega\right)=0, \\
\left|\frac{\partial Y(x, \omega)}{\partial x}\right|_{-L / 2} & =\left|\frac{\partial Y(x, \omega)}{\partial x}\right|_{L / 2}=0,
\end{aligned}
$$

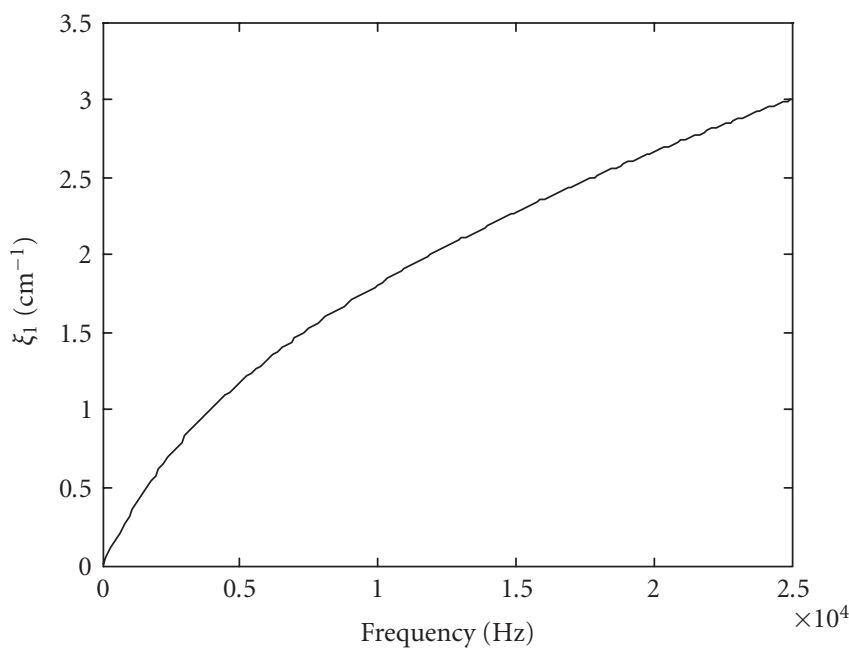

FIgURe 1: Plot of the phase module of the stiff model equation solution for $\varepsilon=\pi / 4 \mathrm{~cm}^{2}$ and $c \approx 2 * 10^{4} \mathrm{~cm} \mathrm{~s}^{-1}$.

and of hinged extrema $[5,16,31,35,36]$ :

$$
\begin{aligned}
Y\left(-\frac{L}{2}, \omega\right) & =Y\left(\frac{L}{2}, \omega\right)=0, \\
\left|\frac{\partial^{2} Y(x, \omega)}{\partial x^{2}}\right|_{-L / 2} & =\left|\frac{\partial^{2} Y(x, \omega)}{\partial x^{2}}\right|_{L / 2}=0 .
\end{aligned}
$$

Before determining the conditions for the eigenfrequencies of the considered stiff systems, we find a more compact way of writing (18) and (19). Starting from the factorized form of the stiff systems equation (see (10)), and using the symbols introduced in Section 2.2, we have

$$
\begin{aligned}
& Y_{1}(x, \omega)=\psi_{1}^{+}(x, \omega)+\psi_{1}^{-}(x, \omega), \\
& Y_{2}(x, \omega)=\psi_{2}^{+}(x, \omega)+\psi_{2}^{-}(x, \omega),
\end{aligned}
$$

where we let

$$
\begin{aligned}
& \psi_{1}^{ \pm}(x, \omega)=c_{1}^{ \pm} \exp \left(\xi_{1}^{ \pm} x\right), \\
& \psi_{2}^{ \pm}(x, \omega)=c_{2}^{ \pm} \exp \left(\xi_{2}^{ \pm} x\right) .
\end{aligned}
$$

Conditions (18) can then be rewritten as follows:

$$
\begin{aligned}
Y_{1}\left(-\frac{L}{2}, \omega\right) & =-Y_{2}\left(-\frac{L}{2}, \omega\right), \\
Y_{1}\left(\frac{L}{2}, \omega\right) & =-Y_{2}\left(\frac{L}{2}, \omega\right), \\
\left|\frac{\partial Y_{1}(x, \omega)}{\partial x}\right|_{-L / 2} & =-\left|\frac{\partial Y_{2}(x, \omega)}{\partial x}\right|_{-L / 2} \\
\left|\frac{\partial Y_{1}(x, \omega)}{\partial x}\right|_{L / 2} & =-\left|\frac{\partial Y_{2}(x, \omega)}{\partial x}\right|_{L / 2} .
\end{aligned}
$$

At the terminations of the string or of the rod, we have

$$
\begin{aligned}
\psi_{1}^{+}+\psi_{1}^{-} & =-\left(\psi_{2}^{+}+\psi_{2}^{-}\right), \\
\xi_{1}^{+} \psi_{1}^{+}+\xi_{1}^{-} \psi_{1}^{-} & =-\left(\xi_{2}^{+} \psi_{2}^{+}+\xi_{2}^{-} \psi_{2}^{-}\right),
\end{aligned}
$$


which can be rewritten in matrix form:

$$
\left[\begin{array}{cc}
1 & 1 \\
\xi_{1}^{+} & \xi_{2}^{+}
\end{array}\right]\left[\begin{array}{l}
\psi_{1}^{+} \\
\psi_{2}^{+}
\end{array}\right]=-\left[\begin{array}{cc}
1 & 1 \\
\xi_{1}^{-} & \xi_{2}^{-}
\end{array}\right]\left[\begin{array}{l}
\psi_{1}^{-} \\
\psi_{2}^{-}
\end{array}\right]
$$

By left-multiplying both members of (24) for the inverse of the $\left[\begin{array}{cc}1 & 1 \\ \xi_{1}^{+} & \xi_{2}^{+}\end{array}\right]$matrix, we have

$$
\left[\begin{array}{l}
\psi_{1}^{+} \\
\psi_{2}^{+}
\end{array}\right]=S_{c}\left[\begin{array}{l}
\psi_{1}^{-} \\
\psi_{2}^{-}
\end{array}\right]
$$

where we let

$$
S_{c} \equiv\left[\begin{array}{cc}
-\frac{\left(\xi_{2}^{+}+\xi_{1}^{+}\right)}{\xi_{2}^{+}-\xi_{1}^{+}} & -2 \frac{\xi_{2}^{+}}{\xi_{2}^{+}-\xi_{1}^{+}} \\
2 \frac{\xi_{1}^{+}}{\xi_{2}^{+}-\xi_{1}^{+}} & \frac{\xi_{2}^{+}+\xi_{1}^{+}}{\xi_{2}^{+}-\xi_{1}^{+}}
\end{array}\right]
$$

The matrix $S_{c}$ relates the incident wave with the reflected wave at the boundaries. Independently of the roots $\xi_{i}$, it has the following properties:

$$
\begin{gathered}
\left|S_{c}\right|=-1, \\
S_{c}^{2}=\left[\begin{array}{ll}
1 & 0 \\
0 & 1
\end{array}\right] .
\end{gathered}
$$

In the case of a hinged stiff system (see (19)) at both ends, we have

$$
\begin{gathered}
\psi_{1}^{+}+\psi_{1}^{-}=-\left(\psi_{2}^{+}+\psi_{2}^{-}\right), \\
\left(\xi_{1}^{+}\right)^{2} \psi_{1}^{+}+\left(\xi_{1}^{-}\right)^{2} \psi_{1}^{-}=-\left(\left(\xi_{2}^{+}\right)^{2} \psi_{2}^{+}+\left(\xi_{2}^{-}\right)^{2} \psi_{2}^{-}\right)
\end{gathered}
$$

which, in matrix form, becomes

$$
\left[\begin{array}{cc}
1 & 1 \\
\left(\xi_{1}^{+}\right)^{2} & \left(\xi_{2}^{+}\right)^{2}
\end{array}\right]\left[\begin{array}{l}
\psi_{1}^{+} \\
\psi_{2}^{+}
\end{array}\right]=-\left[\begin{array}{cc}
1 & 1 \\
\left(\xi_{1}^{-}\right)^{2} & \left(\xi_{2}^{-}\right)^{2}
\end{array}\right]\left[\begin{array}{l}
\psi_{1}^{-} \\
\psi_{2}^{-}
\end{array}\right] .
$$

By taking the inverse of matrix $\left[\begin{array}{cc}1 & 1 \\ \left(\xi_{1}^{+}\right)^{2} & \left(\xi_{2}^{+}\right)^{2}\end{array}\right]$, we obtain

$$
\left[\begin{array}{l}
\psi_{1}^{+} \\
\psi_{2}^{+}
\end{array}\right]=S_{h}\left[\begin{array}{l}
\psi_{1}^{-} \\
\psi_{2}^{-}
\end{array}\right]
$$

where

$$
S_{h}=-\left[\begin{array}{ll}
1 & 0 \\
0 & 1
\end{array}\right]
$$

The $S_{h}$ matrix for the hinged stiff system is independent of roots $\xi_{i}$. The matrices $S_{h}$ and $S_{c}$ are related in the following way:

$$
\begin{aligned}
\left|S_{h}\right| & =-\left|S_{c}\right|, \\
S_{h}^{2} & =S_{c}^{2} .
\end{aligned}
$$

In conclusion, the boundary conditions for stiff systems can be expressed in terms of matrices that can be used in the numerical simulation of stiff systems. Moreover, since the real-life boundary conditions for stiff strings in piano lie in between the conditions given in (18) and (19), we can combine the two matrices $S_{c}$ and $S_{h}$ in order to enforce more general conditions, as illustrated in Section 3. In the following, we will solve (4) and (5) applying separately these sets of boundary conditions.

\subsubsection{The clamped stiff string and rod}

In order to characterize the eigenfunctions in the case of conditions (18), in (12) we let

$$
\xi_{1}=i \xi_{1}^{\prime}
$$

for both the stiff string and the rod solution. By definition, $\xi_{1}^{\prime}$ is a real number. Moreover, for the rod, we have $\xi_{1}^{\prime}=\xi_{2}$. With this position, it can be shown that conditions (18) for the stiff string lead to the equations $[35,38]$

$$
\left[\begin{array}{cc}
\tan \left(\xi_{1}^{\prime} \frac{L}{2}\right) & \tanh \left(\xi_{2} \frac{L}{2}\right) \\
\tanh \left(\xi_{2} \frac{L}{2}\right) & -\tan \left(\xi_{1}^{\prime} \frac{L}{2}\right)
\end{array}\right]\left[\begin{array}{l}
\xi_{1}^{\prime} \\
\xi_{2}
\end{array}\right]=\left[\begin{array}{l}
0 \\
0
\end{array}\right]
$$

while, for the rod, we have

$$
\cos \left(\xi_{1}^{\prime} L\right) \cosh \left(\xi_{2} L\right)=1 .
$$

Equations (34) and (35) can be solved numerically. In particular, taking into account the second line in (12), solutions of (35) are [35]

$$
\begin{aligned}
\omega_{n} & =\frac{\pi^{2}}{4}\left(3.011^{2}, 5^{2}, 7^{2}, \ldots,(2 n+1)^{2}\right) \alpha^{\prime 2}, \\
\alpha^{\prime} & =\frac{\sqrt[4]{\varepsilon^{\prime}}}{L}
\end{aligned}
$$

A similar trend can be obtained for the stiff string. In view of their historical and practical relevance, we here report the numerical approximation for the allowed eigenfrequencies of the stiff string given by Fletcher [27]:

$$
\begin{aligned}
\omega_{n} & \simeq\left(n \pi \frac{c}{L}\right) \sqrt{\left(1+n^{2} \pi^{2} \alpha^{2}\right)}\left(1+2 \alpha+4 \alpha^{2}\right), \\
\alpha & =\frac{\sqrt{\varepsilon}}{L} .
\end{aligned}
$$

If we expand the above expression in a series of powers of $\alpha$ truncated to second order, we have the following approximate formula valid for small values of stiffness:

$$
\omega_{n} \simeq\left(n \pi \frac{c}{L}\right)\left[1+2 \alpha+\left(1+\frac{1}{8} n^{2} \pi^{2}\right)(2 \alpha)^{2}\right] .
$$

The last approximation does not apply to bars. For $\varepsilon=0$, we have $\alpha=0$ and the eigenfrequencies tend to the well-known formula for the vibrating string [35]:

$$
\bar{\omega}_{n}=n \omega_{1} \text {. }
$$

Typical curves of the relative spacing $\chi_{n} \equiv \Delta \omega_{n} / \omega_{1}$, where $\Delta \omega_{n} \equiv \omega_{n+1}-\omega_{n}$, of eigenfrequencies for the stiff string are 


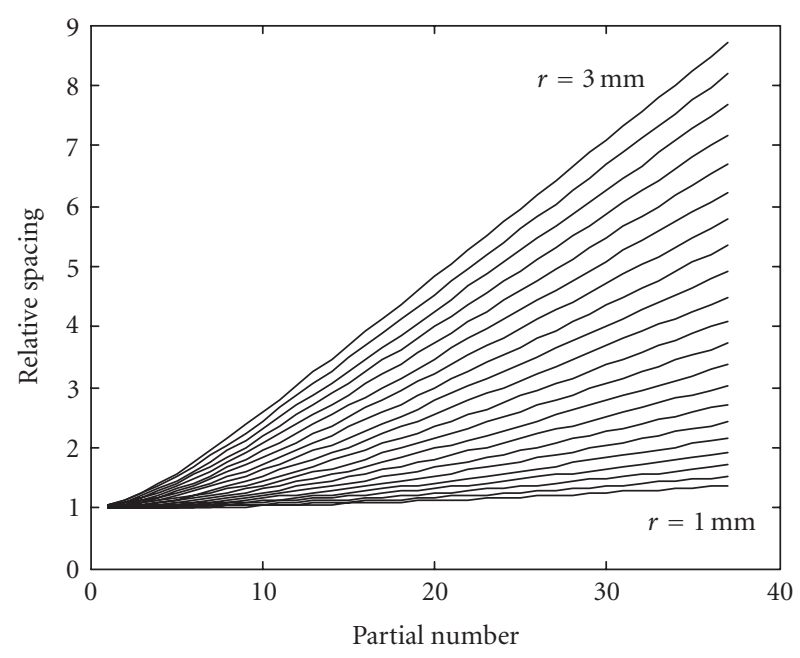

Figure 2: Typical eigenfrequencies relative spacing curves of the clamped stiff string for different values of the radius $r$ of the section $S$.

shown in Figure 2 with variable $r$, where values of the other physical parameters are the same as in (15).

Due to the dependency on the frequency of the phase of the solution, the eigenfrequencies of the stiff string are not equally spaced. For a small radius $r$, hence for low degree of the stiffness of the string (see (1)), the relative spacing is almost constant for all the considered order of eigenfrequencies. However, for higher stiffness, the spacing of the eigenfrequencies increases, in first approximation, as a linear function of the order of the eigenfrequency. The above results are summarized by the typical "warping curves" of the system, shown in Figure 3 , in which the quantity $\omega_{n}-\bar{\omega}_{n}$, which represents the deviation from the linearity, is plotted in terms of spacing $\Delta \omega_{n}$ between consecutive eigenfrequencies.

In the stiff string case, we have two sets of eigenfunctions, one having even parity and the other one having odd parity, whose analytical expressions are respectively given by [38]

$$
\begin{aligned}
& Y(x, \omega)=C(\omega) \cos \left(\xi_{1}^{\prime} \frac{L}{2}\right)\left[\frac{\cos \left(\xi_{1}^{\prime} x\right)}{\cos \left(\xi_{1}^{\prime}(L / 2)\right)}-\frac{\cosh \left(\xi_{2} x\right)}{\cosh \left(\xi_{2}(L / 2)\right)}\right], \\
& Y(x, \omega)=C(\omega) \sin \left(\xi_{1}^{\prime} \frac{L}{2}\right)\left[\frac{\sin \left(\xi_{1}^{\prime} x\right)}{\sin \left(\xi_{1}^{\prime}(L / 2)\right)}-\frac{\sinh \left(\xi_{2} x\right)}{\sinh \left(\xi_{2}(L / 2)\right)}\right],
\end{aligned}
$$

where $C(\omega)$ is a constant that can be calculated imposing the initial conditions.

\subsubsection{Hinged stiff string and rod}

Conditions (19) lead to the following sets of equations for the stiff string:

$$
\begin{gathered}
\sin \left(\xi_{1}^{\prime} L\right) \sinh \left(\xi_{2} L\right)=0, \\
\xi_{1}^{\prime 2}+\xi_{2}^{2}=0,
\end{gathered}
$$

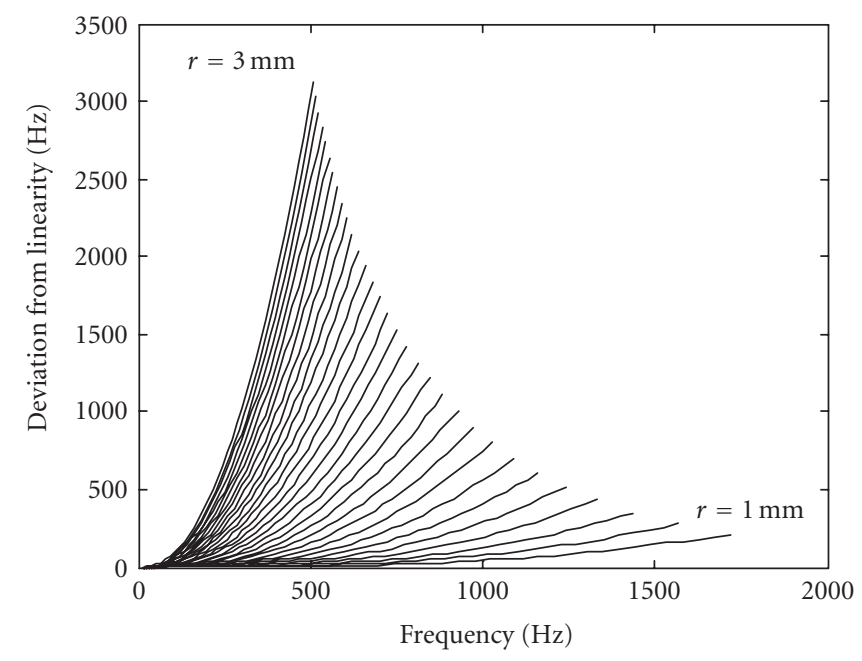

FIGURE 3: Typical warping curves of the clamped stiff string for different values of the radius $r$ of the section $S$.

and for the rod:

$$
\sin \left(\xi_{1}^{\prime} L\right) \sinh \left(\xi_{2} L\right)=0 .
$$

The second line in (41) has no solutions since both $\xi_{1}^{\prime 2}$ and $\xi_{2}^{2}$ are real functions. It follows that hinged stiff systems are only described by (42). In this equation, $\sinh \left(\xi_{2} L\right)=0$ has no solution, hence the eigenfrequencies are determined by the condition

$$
\xi_{1}^{\prime}=\frac{n \pi}{L} .
$$

Using the parameters $\alpha^{\prime}$ and $\alpha$ respectively defined in (36) and (37), the eigenfrequencies for the hinged stiff string are exactly expressed as follows:

$$
\omega_{n}=\left(n \pi \frac{c}{L}\right) \sqrt{\left(n^{2} \pi^{2} \alpha^{2}+1\right)},
$$

while for the rod, we have

$$
\omega_{n}=n^{2} \pi^{2} \alpha^{\prime 2} .
$$

As the tension $T \rightarrow 0,(44)$ tends to (45). Figure 4 shows the relative spacing of the eigenfrequencies in the case of the hinged stiff string.

Relative eigenfrequencies spacing curves are very similar to the ones of the clamped string and so are the "warping curves" of the system, as shown in Figure 5.

Using (45), we can give an analytic expression for the relative spacing of the eigenfrequencies of the hinged rod. We have

$$
\pi^{2} \alpha^{\prime 2}(2 n+1)
$$

Equation (43) leads to the following set of odd and even 


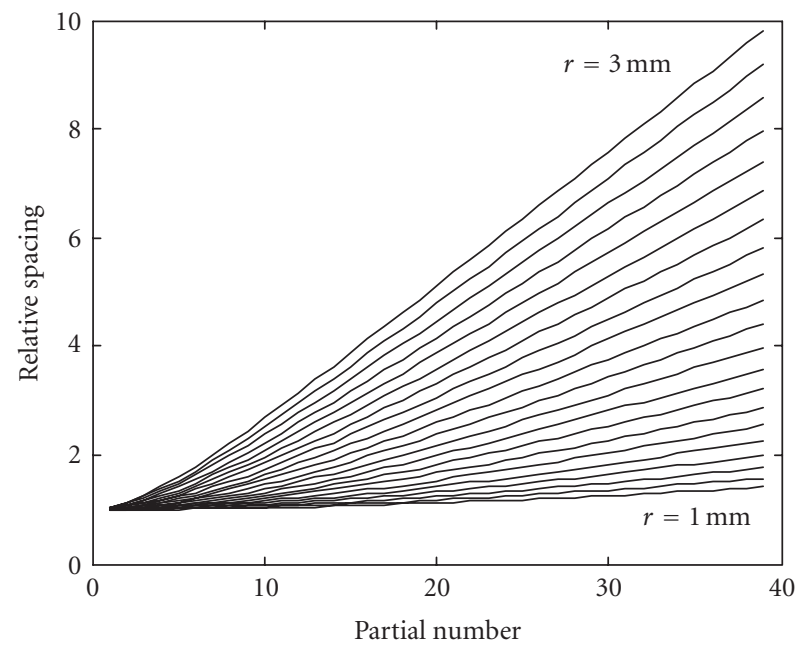

Figure 4: Typical eigenfrequencies relative spacing curves of the hinged stiff string for different values of the radius $r$ of the section S.

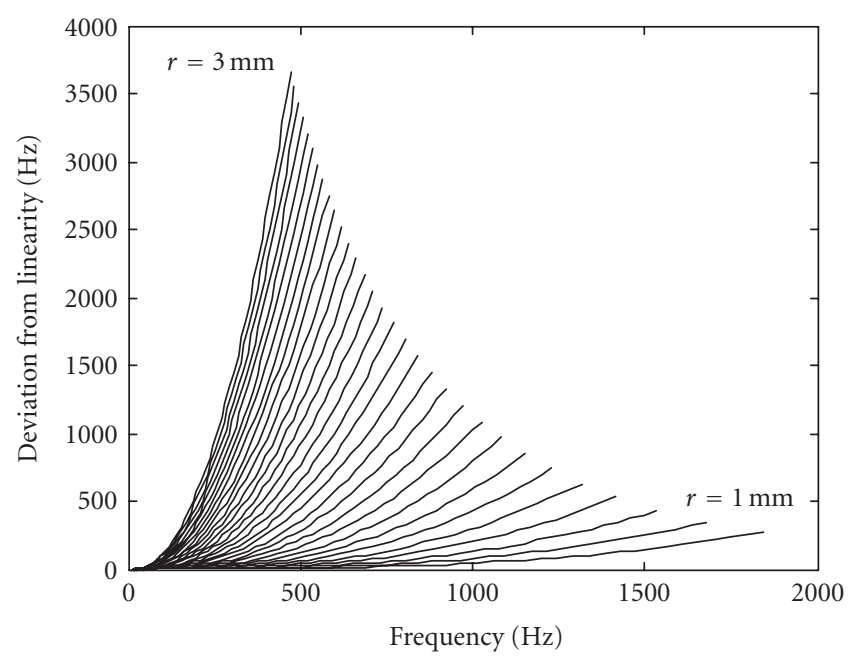

FIGURE 5: Typical warping curves of the hinged stiff string for different values of the radius $r$ of the section $S$.

eigenfunctions for the stiff string [38]:

$$
\begin{aligned}
& Y_{n}(x, \omega)=2 D(\omega) \sin \left(\frac{2 n \pi}{L} x\right), \\
& Y_{n}(x, \omega)=2 D(\omega) \cos \left(\frac{(2 n+1) \pi}{L} x\right),
\end{aligned}
$$

where $D(\omega)$ must be determined by enforcing the initial conditions. It is worth noting that both functions in (47) are independent of the stiffness parameter $\varepsilon$. In Section 3, we will use the obtained results in order to implement the dispersive waveguides digitally simulating the solutions of (4) and (5).

Finally, we need to stress the fact that the eigenfrequencies of the hinged stiff string are similar to the ones for the clamped case except for the factor $\left(1+2 \alpha+4 \alpha^{2}\right)$. Therefore, for small values of stiffness, they do not differ too much. This

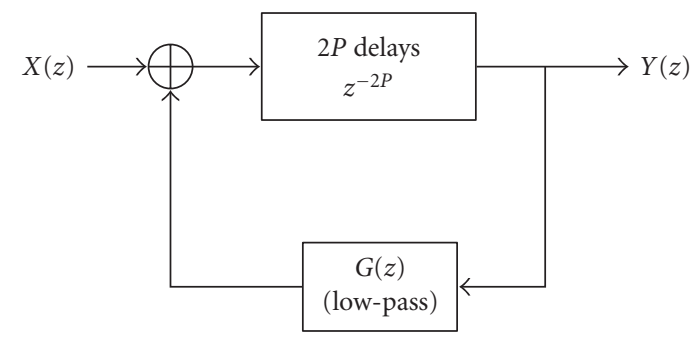

Figure 6: Basic Karplus-Strong delays cascade.

can also be seen from the similarity of the warping curves obtained with the two types of boundary conditions.

Taking into account the fact that real-piano strings boundary conditions lie in between these two cases, we can conclude that the eigenfrequencies of real-piano strings can be calculated by means of the approximated formula $[27,28]$ :

$$
\omega_{n} \simeq A n \sqrt{B n^{2}+1},
$$

where $A$ and $B$ can be obtained from measurements. Approximation (48) is useful in order to match measured vibrating modes against the model eigenfrequencies.

\section{NUMERICAL APPROXIMATIONS OF STIFF SYSTEMS}

Most of the problems encountered when dealing with the continuous-time equation of the stiff string consist in determining the general solution and in relating the initial and boundary conditions to the integrating constants of the equation. In this section, we will show that we can use a similar technique also in discrete-time, which yields a numerical transform method for the computation of the solution.

In Section 2, we noted that (1) becomes the equation of vibrating string in the case of negligible stiffness coefficient $\varepsilon$. It is well known that the technique known as Karplus-Strong algorithm implements the discrete-time domain solution of the vibrating string equation [8], allowing us to reach good quality acoustic results. The block diagram of the adopted loop circuit is shown in Figure 6.

The transfer function of the digital loop chain can be written as follows:

$$
H(z)=\frac{1}{1-z^{-2 P} G(z)},
$$

where the loop filter $G(z)$ takes into account losses due to nonrigid terminations and to internal friction, and $P$ is the number of sections in which the string is subdivided, as obtained from time and space sampling. Loop filters design can be based on measured partial amplitude and frequency trajectories [18], or on linear predictive coding (LPC)-type methods [9]. The filter $G(z)$ can be modelled as IIR or FIR and it must be estimated from samples of the sound or from a model of the string losses, where, for stability, we need $\left|G\left(e^{j \omega}\right)\right|<1$. Clearly, in the IIR case or in the nonlinear phase FIR case, the phase response of the loop filter introduces a 


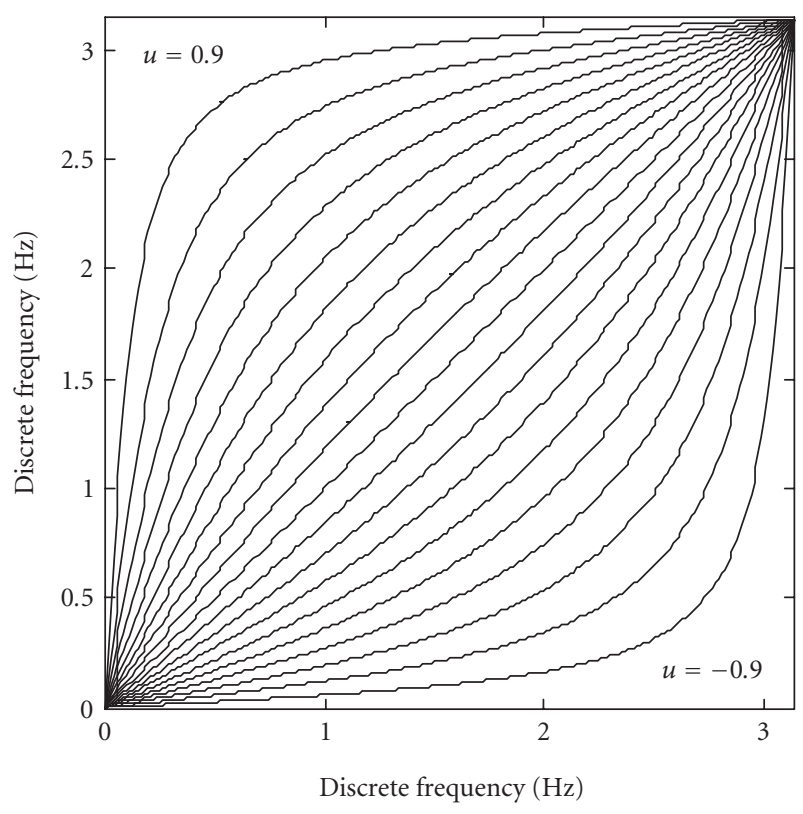

FIGURE 7: First-order all-pass phase plotted for various values of $u$.

limited amount of dispersion. Additional phase terms in the form of all-pass filters can be added in order to tune the string model to the required pitch [13] and contribute to further dispersion.

Since the group velocity for a traveling wave for a stiff system depends on frequency (see (16)), it is natural to substitute, in discrete time, the cascade of unit delays with a chain of circuital elements whose phase responses do depend on frequency. One can show that the only choice that leads to rational transfer functions is given by a chain of first-order all-pass filters $[39,40]$. More complex physical systems, for example, as in the simulation of a monaural room, call for substituting the delays chain with a more general filter as illustrated in [41]:

$$
A(z, u)=\frac{z^{-1}-u}{1-u z^{-1}}
$$

whose phase characteristic is

$$
\theta(\Omega)=\Omega+2 \arctan \frac{u \sin (\Omega)}{1-u \cos (\Omega)}
$$

The phase characteristics in (51) are plotted in Figure 7 for various values of $u$.

A comparison between the curve in Figure 1 and the ones in Figure 7 gives more elements of plausibility for the approximation of the solution phase of the stiff model equations, given in (12), with the all-pass filter phase (51). Adopting a similar circuital scheme as in the Karplus-String algorithm [10] in which the unit delays are replaced by first-order allpass filters, the approximation is given by

$$
\xi_{1}^{\prime}\left(\Omega f_{s}\right) \simeq \frac{P}{L} \theta(\Omega)
$$

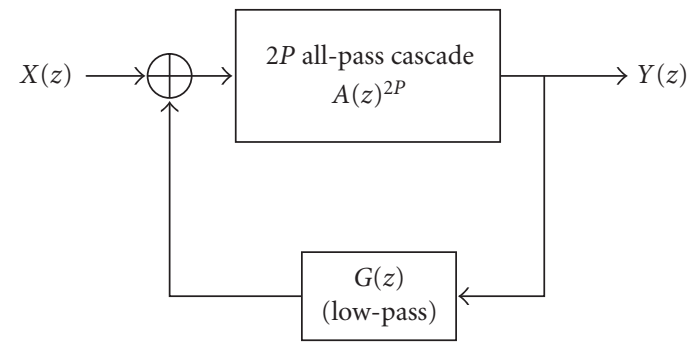

FIGURE 8: Dispersive waveguide used to simulate dispersive systems.

where $f_{s}$ is the sampling frequency. Note that, by definition, both members of (52) are real numbers. Therefore, in the $z$ domain, a nonstiff system can be mapped into a stiff system by means of the frequency warping map

$$
z^{-1} \longrightarrow A(z)
$$

The resulting circuit is shown in Figure 8. Note, that the feedback all-pass chain results in delay-free loops. Computationally, these loops can be resolved by the methods illustrated in $[34,42,43]$. Moreover, the phase response of the loop filter $G(z)$ contributes to the dispersion and it must be taken into account in the global model.

The circuit in Figure 8 can be optimized in order to take into account the losses and the coupling amongst strings (e.g., as in piano). In the framework of this paper, we confined our interest to the design of the stiff system filter. For a review of the design of lossy filters and coupling models, see [17].

\subsection{Stiff system filter parameters determination}

Within the framework of the approximation (52) in the case of dispersive waveguide, the integer parameter $P$ can be obtained by constraining the two functions to attain the same values at the extrema of the bandwidth. Since $\theta(\pi)=\pi$, we have

$$
P=\frac{\xi_{1}\left(\pi f_{s}\right) L}{\pi}
$$

As we will see, condition (54) is not the only one that can be obtained for the parameter $P$. The deviation from linearity introduced by the warping $\theta(\Omega)$ can be written as follows:

$$
\Delta(\Omega) \equiv \theta(\Omega)-\Omega=2 \arctan \frac{u \sin (\Omega)}{1-u \cos (\Omega)} .
$$

The function $\Delta(\Omega)$ is plotted, for different values of $u$, in Figure 9.

One can see that the absolute value of $\Delta(\Omega)$ has a maximum which corresponds to the maximum deviation from the linearity of $\theta(\Omega)$. It can be shown that this maximum occurs for

$$
\Omega=\Omega_{M}=\arccos (u)
$$




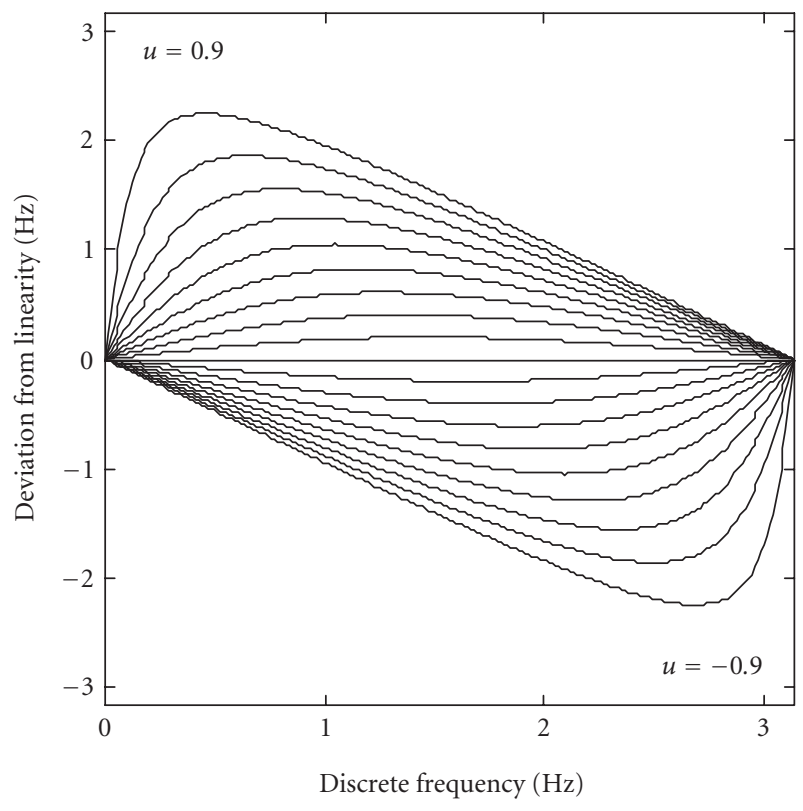

FIgURE 9: Plot of the deviation from linearity of the all-pass filter phase for different values of parameter $u$.

for which the maximum deviation is

$$
\Delta\left(\Omega_{M}, u\right)=2 \arcsin (u) .
$$

Substituting (56) in (51), we have

$$
\theta\left(\Omega_{M}\right)=\frac{\pi}{2}+\arcsin (u)
$$

Since the solution phase $\xi_{1}$ is approximated by $\theta(\Omega)$, it has to satisfy the condition

$$
\xi_{1}\left(\frac{\Omega_{M}}{T}\right) \frac{L}{P} \leqslant \frac{\pi}{2}+\arcsin (u)
$$

and therefore, we have the following bound on $P$ :

$$
P \geqslant \frac{L \xi_{1}\left(f_{s} \arccos (u)\right)}{\pi / 2+\arcsin (u)} .
$$

For higher-order $Q$ all-pass filters, (60) can be written as follows:

$$
P \geqslant \frac{1}{Q} \sum_{i=1}^{Q} \frac{\xi_{1}\left(f_{s} \arccos \left(u_{i}\right)\right) L}{\pi / 2+\arcsin \left(u_{i}\right)} .
$$

An optimization algorithm can be used to obtain the vector parameter $\mathbf{u}$. Based on our experiments, we estimated that an optimal order $Q$ is 4 for the piano string. Therefore, using the values in (15) for the $58 \mathrm{~Hz}$ tone of an $L=200 \mathrm{~cm}$ brass string, we obtain $P=209$. Although this is not a model for a real-life wound inhomogeneous piano string, this example gives a rough idea of the typical number of the required all-pass sections. The computation of this long allpass chain can be too heavy for real-time applications. There- fore, an approximation of the chain by means of a cascade of an all-pass of order much smaller than $2 P$ with unit delays is usually sought $[13,29,30]$. A simple and accurate approach is to model the all-pass as a cascade of first-order sections with variable real parameter $u$ [38]. However, a more general approach calls for including in the design second-order all-pass sections, equivalent to a pair of complex conjugated first-order sections [29]. In Section 4, we will bypass this estimation procedure based on the theoretical eigenfunctions of the string to estimate the all-pass parameters and the number of sections from samples of the piano.

\subsection{Laguerre sequences}

An invertible and orthogonal transform, which is related to the all-pass chain included in the stiff string model, is given by the Laguerre transform $[44,45]$. The Laguerre sequences $l_{i}[m, u]$ are best defined in the $z$-domain as follows:

$$
L_{i}(z, u)=\frac{\sqrt{1-u^{2}}}{1-u z^{-1}}\left[\frac{z^{-1}-u}{1-u z^{-1}}\right]^{i} .
$$

Thus, the Laguerre sequences can be obtained from the $z$ domain recurrence

$$
\begin{aligned}
L_{0}(z, u) & =\frac{\sqrt{1-u^{2}}}{1-u z^{-1}}, \\
L_{i+1}(z, u) & =A(z) L_{i}(z, u),
\end{aligned}
$$

where $A(z)$ is defined as in (50). Comparison of (62) with (50) shows that the phase of the $z$ transform of the Laguerre sequences is suitable for approximating the phase of the solution of the stiff model equation. A biorthogonal generalization of the Laguerre sequences calling for a variable $u$ from section to section is illustrated in [46]. This is linked to the refined approximation of the solution previously shown.

\subsection{Initial conditions}

Putting together the results obtained in Section 1, we can write the solution phase of the stiff model $Y(\Omega, x)$ as follows (see (11) and (14)):

$$
Y(\omega, x)=c_{1}^{+}(\omega) \exp \left(i \xi_{1}^{\prime} x\right)+c_{1}^{-}(\omega) \exp \left(-i \xi_{1}^{\prime} x\right) .
$$

We are now disregarding the transient term due to $\xi_{2}$ since it does not influence the acoustic frequencies of the system. In discrete time and space, we let $x=m(L / P)$ as in [10]. With the approximation (52), (64) becomes

$$
Y(m, \Omega) \simeq c_{1}^{+}(\Omega) \exp (i m \theta(\Omega))+c_{1}^{-}(\Omega) \exp (-i m \theta(\Omega)) .
$$

Substituting (63) in (65), we have

$$
Y(\Omega, m) \simeq c_{1}^{+}(\Omega) \frac{L_{m}(\Omega, u)}{L_{0}(z, u)}+c_{1}^{-}(\Omega) \frac{L_{-m}(\Omega, u)}{L_{0}(z, u)},
$$

where we have used the fact that

$$
A\left(e^{i \Omega}, u\right)=\frac{e^{-i \Omega}-u}{1-u e^{-i \Omega}}=\exp (i \theta(\Omega)) .
$$


By defining

$$
V_{+}(\Omega) \equiv \frac{c_{1}^{+}(\Omega)}{L_{0}(z, u)}, \quad V_{-}(\Omega) \equiv \frac{c_{1}^{-}(\Omega)}{L_{0}(z, u)},
$$

(66) can be written as follows:

$$
Y(m, \Omega) \simeq V_{+}(\Omega) L_{m}(\Omega, u)+V_{-}(\Omega) L_{-m}(\Omega, u) .
$$

Taking the inverse discrete-time Fourier transform (IDTFT) on both sides of (69), we obtain

$$
y[m, n] \simeq y_{+}[m, n]+y_{-}[m, n],
$$

where

$$
\begin{aligned}
& y_{+}[m, n]=\sum_{k=-\infty}^{\infty} v_{+}[n-k] l_{m}[k, u], \\
& y_{-}[m, n]=\sum_{k=-\infty}^{\infty} v_{-}[n-k] l_{-m}[k, u],
\end{aligned}
$$

and the sequences $v_{ \pm}(n)$ are the IDTFT of $V_{ \pm}(\Omega)$. For the sake of conciseness, we do not report here the expression of $v_{ \pm}[n]$ in terms of constants $c_{1}^{ \pm}$. For further details, see [31, $38]$. The expression of the numerical solution $y[m, n]$ can be written in terms of a generic initial condition

$$
y[m, 0]=y_{+}[m, 0]+y_{-}[m, 0] .
$$

In order to do this, we resort to the extension of Laguerre sequences to negative arguments:

$$
l_{m}[n, u]= \begin{cases}l_{m}[n, u], & n \geq 0, \\ l_{m}[-n, u], & n<0,\end{cases}
$$

and to the property

$$
l_{m}[n, u]=l_{n}[m,-u] .
$$

If we introduce the quantity

$$
\begin{gathered}
y_{k}^{ \pm}[u]=\sum_{m=0}^{\infty} y_{ \pm}[m, 0] l_{k}^{\top}[ \pm m, u], \\
l_{k}^{\top}[ \pm m, u]=l_{ \pm m}[k, u],
\end{gathered}
$$

with a simple mathematical manipulation, (71) can be written as follows:

$$
\begin{aligned}
& y_{+}[m, n]=\sum_{k=-\infty}^{\infty} y_{k}^{+}[u] l_{m}[k+n, u], \\
& y_{-}[m, n]=\sum_{k=-\infty}^{\infty} y_{k}^{-}[u] l_{m}[k+n, u] .
\end{aligned}
$$

Therefore, the numeric solution becomes

$$
y[m, n]=\sum_{k=-\infty}^{\infty} y_{k}^{+} l_{m}[k+n, u]+\sum_{k=-\infty}^{\infty} y_{k}^{-} l_{m}[k+n, u] .
$$

We have just shown that the solution of the discrete-time stiff model equation can be written as a Laguerre expansion of the initial condition. At the same time, this shows that the stiff string model is equivalent to a nonstiff string model cascaded by frequency warping obtained by Laguerre expansion.

\subsection{Boundary conditions}

In Section 1, we discussed the stiff model equation boundary conditions in continuous time (see (18) and (19)). In this section, we will discuss the homogenous boundary conditions (i.e., the first line in both (18) and (19)) in the discrete-time domain. Using approximation (52) and letting the number of sections of the stiff system $P$ be an even integer, we can write the homogenous conditions as follows (see also (69)):

$$
\begin{aligned}
& Y\left(-\frac{P}{2}, \Omega\right)=0 \\
& \quad \Longrightarrow V_{+}(\Omega) L_{-P / 2}(\Omega, u)+V_{-}(\Omega) L_{P / 2}(\Omega, u)=0 \\
& Y\left(+\frac{P}{2}, \Omega\right)=0 \\
& \quad \Longrightarrow V_{+}(\Omega) L_{P / 2}(\Omega, u)+V_{-}(\Omega) L_{-P / 2}(\Omega, u)=0 .
\end{aligned}
$$

Like (34), (78) can be expressed in matrix form:

$$
\left[\begin{array}{cc}
L_{P / 2}(\Omega, u) & L_{-P / 2}(\Omega, u) \\
L_{-P / 2}(\Omega, u) & L_{P / 2}(\Omega, u)
\end{array}\right]\left[\begin{array}{c}
V_{+}(\Omega) \\
V_{-}(\Omega)
\end{array}\right]=\left[\begin{array}{l}
0 \\
0
\end{array}\right] .
$$

As shown in Section 3.3, the functions $V_{ \pm}(\Omega)$ are determined by means of Laguerre expansion of the initial conditions sequences through (71) and (76). For any choice of these initial conditions, the determinant of the coefficients matrix in (79) must be zero, obtaining the following condition:

$$
\left[L_{P / 2}(\Omega, u)\right]^{2}-\left[L_{-P / 2}(\Omega, u)\right]^{2}=0 .
$$

Recalling the $z$-transform expression for the Laguerre sequences, we have

$$
\sin [\theta(\Omega) P]=0, \quad \theta(\Omega)=\frac{k \pi}{P}, \quad k=1,2,3, \ldots
$$

In the stiff string case, the eigenfrequencies of the system are not harmonically related. In our approximation of the phase of the solution with the digital all-pass phase, the harmonicity is reobtained at a different level: the displacement of the all-pass phase values is harmonic according to the law written in (81). The distance between two consecutive values of this phase is $\pi / P$. Due to the nonrigid terminations, the reallife boundary conditions can be given in terms of frequency dependent functions, which are included in the loop filter. In mapping the stiff structure to a nonstiff one, care must be taken into unwarping the loop filter as well.

\section{SYNTHESIS OF SOUND}

In order to implement a piano simulation via the physical model, we need to determine the design parameters of the 


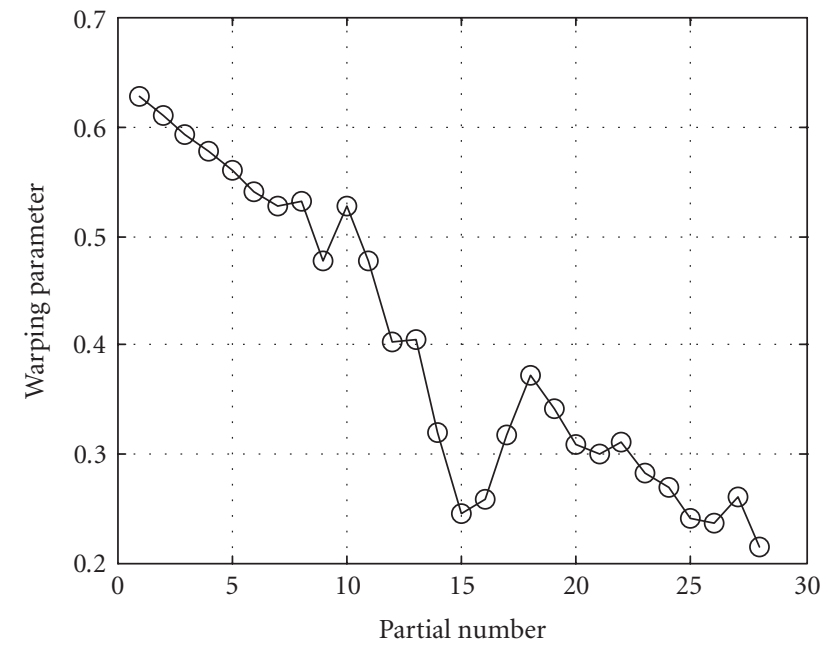

FIgURE 10: Computed all-pass optimized parameters $u$.

dispersive waveguide, that is, the number of all-pass sections and the coefficients $u_{i}$ of the all-pass filters. This task could be performed by means of lengthy measurements or estimation of the physical variables, such as tension, Young's module, density, and so forth. However, as we already remarked, due to the constitutive complexity of the real-life piano strings and terminations, this task seems to be quite difficult and to lead to inaccurate results. In fact, the given physical model only approximately matches the real situation. Indeed, in order to model and justify the measured eigenfrequencies, we resorted to Fletcher's experimental model described by (48). However, in that case, we ignore the exact form of the eigenfunctions, which is required in order to determine the number of sections of the waveguide and the other parameters. A more pragmatic and effective approach is to estimate the waveguide parameters directly from the measured eigenfrequencies $\omega_{n}$. These can be extracted, for example, from recorded samples of notes played by the piano under exam. Fletcher's parameters $A$ and $B$ can be calculated as follows:

$$
\begin{aligned}
& A=\frac{1}{2 n} \sqrt{\frac{16 \omega_{n}^{2}-\omega_{2 n}^{2}}{3}}, \\
& B=\frac{1}{n^{2}} \frac{4 \gamma^{2}-1}{1-16 \gamma^{2}}, \quad \gamma=\frac{\omega_{n}}{\omega_{2 n}} .
\end{aligned}
$$

In practice, in the model where the all-pass parameters $u_{i}$ are equal throughout the delay line, one does not even need to estimate Fletcher's parameters. In fact, in view of the equivalence of the stiff string model with the warped nonstiff model, one can directly determine, through optimization, the parameter $u$ that makes the dispersion curve of the eigenfrequencies the closest to a straight line, using a suitable distance. A result of this optimization is shown in Figure 10. It must be pointed out that our point of view differs from the one proposed in $[29,30]$, where the objective is the min-

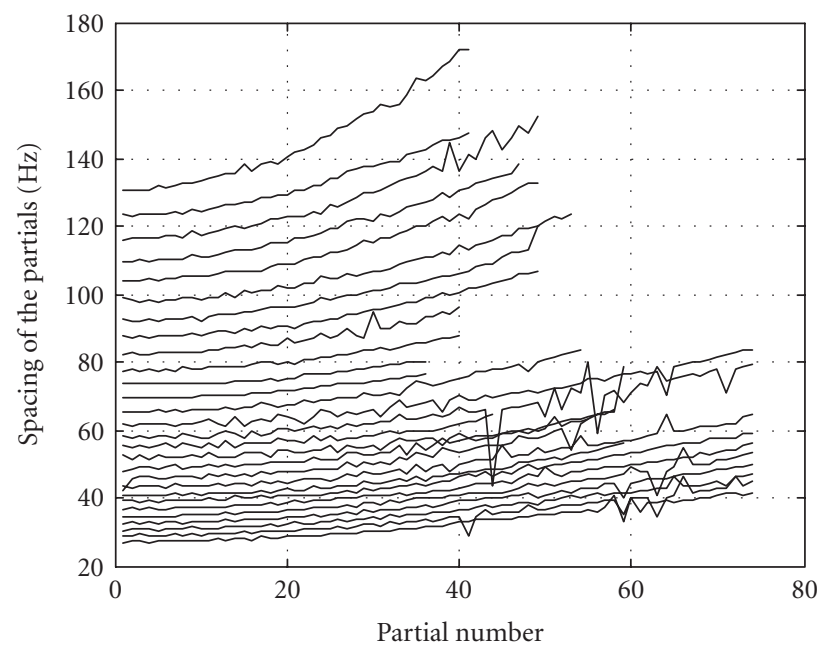

FiguRE 11: Warped deviation from linearity.

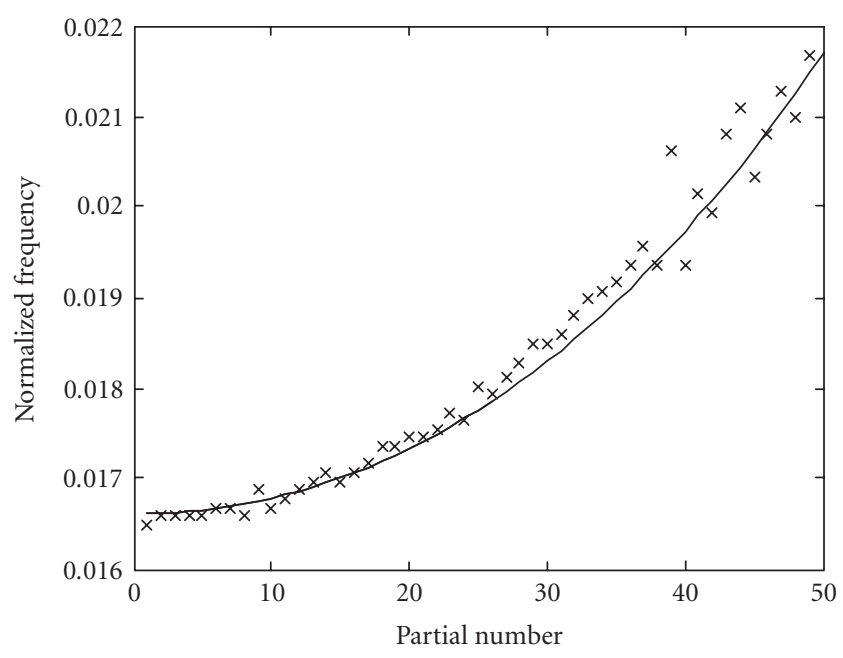

FIgURE 12: Optimized all-pass parameters $u$ for A\#3 tone.

imization of the number of nontrivial all-pass sections in the cascade.

Given the optimum warping curve, the number of sections is then determined by forcing the pitch of the cascade of the nonstiff model (Karplus-Strong like) with warping to match the required fundamental frequency of the recorded tone. An example of this method is shown in Figure 11, where the measured warping curves pertaining to several piano keys in the low register, as estimated from the resonant eigenfrequencies, are shown. In Figure 12, the optimum sequence of all-pass parameters $u$ for the examined tones is shown. Finally, in Figure 13, the plot of the regularized dispersion curves by means of optimum unwarping is shown. For further details about this method, see [47, 48, 49]. Frequency warping has also been employed in conjunction with $2 \mathrm{D}$ waveguide meshes in the effort of reducing the artificial 


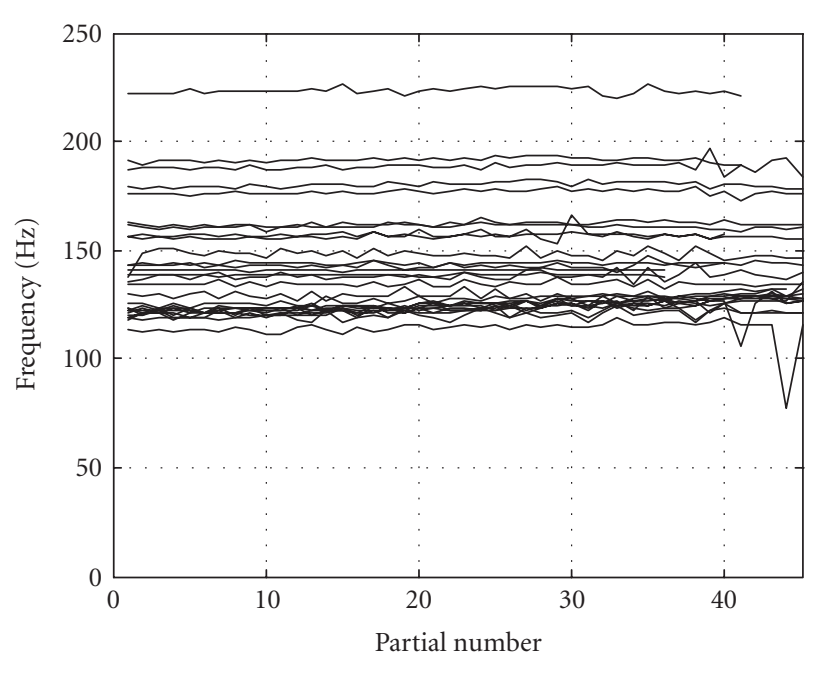

FIgURE 13: Optimum unwarped regularized dispersion curves.

dispersion introduced by the nonisotropic spatial sampling [50]. Since the required warping curves do not match the first-order all-pass phase characteristic, in order to overcome this difficulty, a technique including resampling operators has been used in $[50,51]$ according to a scheme first introduced in [33] and further developed in [52] for the wavelet transforms. However, the downsampling operators inevitably introduce aliasing. While in the context of wavelet transforms, this problem is tackled with multichannel filter banks, this is not the case of $2 \mathrm{D}$ waveguide meshes.

\section{CONCLUSIONS}

In order to support the design and use of digital dispersive waveguides, we reviewed the physical model of stiff systems, using a frequency domain approach in both continuous and discrete time. We showed that, for dispersive propagation in the discrete-time, the Laguerre transform allows us to write the solution of the stiff model equation in terms of an orthogonal expansion of the initial conditions and to reobtain harmonicity at the level of the displacement of the allpass phase values. Consequently, we showed that the stiff string model is equivalent to a nonstiff string model cascaded with frequency warping, in turn obtained by Laguerre expansion. Finally, we showed that due to this equivalence, the all-pass coefficients can be computed by means of optimization algorithms of the stiff model with a warped nonstiff one.

The exploration of physical models of musical instruments requires mathematical or physical approximations in order to make the problem treatable. When available, the solutions will only partially reflect the ensemble of mechanical and acoustic phenomena involved. However, the physical models serve as a solid background for the construction of physically inspired models, which are flexible numerical approximations of the solutions. Per se, these approximations are interesting for the synthesis of virtual in- struments. However, in order to fine tune the physically inspired models to real instruments, one needs methods for the estimation of the parameters from samples of the instrument. In this paper, we showed that dispersion from stiffness is a simple case in which the solution of the raw physical model suggests a discrete-time model, which is flexible enough to be used in the synthesis and which provides realistic results when the characteristics are estimated from the samples.

\section{REFERENCES}

[1] B. L. Vercoe, W. G. Gardner, and E. D. Scheirer, "Structured audio: creation, transmission, and rendering of parametric sound representations," Proceedings of the IEEE, vol. 86, no. 5, pp. 922-940, 1998.

[2] P. Cook, "Physically informed sonic modeling (PhISM): synthesis of percussive sounds," Computer Music Journal, vol. 21, no. 3, pp. 38-49, 1997.

[3] L. Hiller and P. Ruiz, "Synthesizing musical sounds by solving the wave equation for vibrating objects: Part I," Journal of the Audio Engineering Society, vol. 19, no. 6, pp. 462-470, 1971.

[4] L. Hiller and P. Ruiz, "Synthesizing musical sounds by solving the wave equation for vibrating objects: Part II," Journal of the Audio Engineering Society, vol. 19, no. 7, pp. 542-551, 1971.

[5] A. Chaigne and A. Askenfelt, "Numerical simulations of piano strings. I. A physical model for a struck string using finite difference methods," Journal of the Acoustical Society of America, vol. 95, no. 2, pp. 1112-1118, 1994.

[6] A. Chaigne and A. Askenfelt, "Numerical simulations of piano strings. II. Comparisons with measurements and systematic exploration of some hammer-string parameters," Journal of the Acoustical Society of America, vol. 95, no. 3, pp. 1631-1640, 1994.

[7] A. Chaigne, "On the use of finite differences for musical synthesis. Application to plucked stringed instruments," Journal d'Acoustique, vol. 5, no. 2, pp. 181-211, 1992.

[8] D. A. Jaffe and J. O. Smith III, "Extensions of the KarplusStrong plucked-string algorithm," The Music Machine, C. Roads, Ed., pp. 481-494, MIT Press, Cambridge, Mass, USA, 1989.

[9] J. O. Smith III, Techniques for digital filter design and system identification with application to the violin, Ph.D. thesis, Electrical Engineering Department, Stanford University (CCRMA), Stanford, Calif, USA, June 1983.

[10] K. Karplus and A. Strong, "Digital synthesis of plucked-string and drum timbres," The Music Machine, C. Roads, Ed., pp. 467-479, MIT Press, Cambridge, Mass, USA, 1989.

[11] J. O. Smith III, "Physical modeling using digital waveguides," Computer Music Journal, vol. 16, no. 4, pp. 74-91, 1992.

[12] J. O. Smith III, "Physical modeling synthesis update," Computer Music Journal, vol. 20, no. 2, pp. 44-56, 1996.

[13] S. A. Van Duyne and J. O. Smith III, "A simplified approach to modeling dispersion caused by stiffness in strings and plates," in Proc. 1994 International Computer Music Conference, pp. 407-410, Aarhus, Denmark, September 1994.

[14] J. O. Smith III, "Principles of digital waveguide models of musical instruments," in Applications of Digital Signal Processing to Audio and Acoustics, M. Kahrs and K. Brandenburg, Eds., pp. 417-466, Kluwer Academic Publishers, Boston, Mass, USA, 1998.

[15] M. Karjalainen, T. Tolonen, V. Välimäki, C. Erkut, M. Laurson, and J. Hiipakka, "An overview of new techniques and effects in model-based sound synthesis," Journal of New Music Research, vol. 30, no. 3, pp. 203-212, 2001. 
[16] J. Bensa, S. Bilbao, R. Kronland-Martinet, and J. O. Smith III, "The simulation of piano string vibration: from physical models to finite difference schemes and digital waveguides," Journal of the Acoustical Society of America, vol. 114, no. 2, pp. 1095-1107, 2003.

[17] B. Bank, F. Avanzini, G. Borin, G. De Poli, F. Fontana, and D. Rocchesso, "Physically informed signal processing methods for piano sound synthesis: a research overview," EURASIP Journal on Applied Signal Processing, vol. 2003, no. 10, pp. 941-952, 2003.

[18] V. Välimäki, J. Huopaniemi, M. Karjalainen, and Z. Jánosy, "Physical modeling of plucked string instruments with application to real-time sound synthesis," Journal of the Audio Engineering Society, vol. 44, no. 5, pp. 331-353, 1996.

[19] J. O. Smith III, "Efficient synthesis of stringed musical instruments," in Proc. 1993 International Computer Music Conference, pp. 64-71, Tokyo, Japan, September 1993.

[20] M. Karjalainen, V. Välimäki, and Z. Jánosy, "Towards highquality sound synthesis of the guitar and string instruments," in Proc. 1993 International Computer Music Conference, pp. 56-63, Tokyo, Japan, September 1993.

[21] G. Borin and G. De Poli, "A hysteretic hammer-string interaction model for physical model synthesis," in Proc. Nordic Acoustical Meeting, pp. 399-406, Helsinki, Finland, June 1996.

[22] G. E. Garnett, "Modeling piano sound using digital waveguide filtering techniques," in Proc. 1987 International Computer Music Conference, pp. 89-95, Urbana, Ill, USA, August 1987.

[23] J. O. Smith III and S. A. Van Duyne, "Commuted piano synthesis," in Proc. 1995 International Computer Music Conference, pp. 319-326, Banff, Canada, September 1995.

[24] S. A. Van Duyne and J. O. Smith III, "Developments for the commuted piano," in Proc. 1995 International Computer Music Conference, pp. 335-343, Banff, Canada, September 1995.

[25] M. Karjalainen and J. O. Smith III, "Body modeling techniques for string instrument synthesis," in Proc. 1996 International Computer Music Conference, pp. 232-239, Hong Kong, August 1996.

[26] M. Karjalainen, V. Välimäki, and T. Tolonen, "Plucked-string models, from the Karplus-Strong algorithm to digital waveguides and beyond," Computer Music Journal, vol. 22, no. 3, pp. 17-32, 1998.

[27] H. Fletcher, "Normal vibration frequencies of a stiff piano string," Journal of the Acoustical Society of America, vol. 36, no. 1, pp. 203-209, 1964.

[28] H. Fletcher, E. D. Blackham, and R. Stratton, "Quality of piano tones," Journal of the Acoustical Society of America, vol. 34, no. 6, pp. 749-761, 1962.

[29] D. Rocchesso and F. Scalcon, "Accurate dispersion simulation for piano strings," in Proc. Nordic Acoustical Meeting, pp. 407414, Helsinki, Finland, June 1996.

[30] D. Rocchesso and F. Scalcon, "Bandwidth of perceived inharmonicity for physical modeling of dispersive strings," IEEE Trans. Speech and Audio Processing, vol. 7, no. 5, pp. 597-601, 1999.

[31] I. Testa, G. Evangelista, and S. Cavaliere, "A physical model of stiff strings," in Proc. Institute of Acoustics (Internat. Symp. on Music and Acoustics), vol. 19, pp. 219-224, Edinburgh, UK, August 1997.

[32] S. Cavaliere and G. Evangelista, "Deterministic least squares estimation of the Karplus-Strong synthesis parameter," in Proc. International Workshop on Physical Model Synthesis, pp. 15-19, Firenze, Italy, June 1996.

[33] G. Evangelista and S. Cavaliere, "Discrete frequency warped wavelets: theory and applications," IEEE Trans. Signal Processing, vol. 46, no. 4, pp. 874-885, 1998.
[34] A. Härmä, M. Karjalainen, L. Savioja, V. Välimäki, U. K. Laine, and J. Huopaniemi, "Frequency-warped signal processing for audio applications," Journal of the Audio Engineering Society, vol. 48, no. 11, pp. 1011-1031, 2000.

[35] N. H. Fletcher and T. D. Rossing, Principles of Vibration and Sound, Springer-Verlag, New York, NY, USA, 1995.

[36] L. D. Landau and E. M. Lifšits, Theory of Elasticity, Editions Mir, Moscow, Russia, 1967.

[37] N. Dunford and J. T. Schwartz, Linear Operators. Part 2: Spectral Theory, Self Adjoint Operators in Hilbert Space, John Wiley \& Sons, New York, NY, USA, 1st edition, 1963.

[38] I. Testa, Sintesi del suono generato dalle corde vibranti: un algoritmo basato su un modello dispersivo, Physics degree thesis, Università Federico II di Napoli, Napoli, Italy, 1997.

[39] H. W. Strube, "Linear prediction on a warped frequency scale," Journal of the Acoustical Society of America, vol. 68, no. 4, pp. 1071-1076, 1980.

[40] J. A. Moorer, "The manifold joys of conformal mapping: applications to digital filtering in the studio," Journal of the Audio Engineering Society, vol. 31, no. 11, pp. 826-841, 1983.

[41] J.-M. Jot and A. Chaigne, "Digital delay networks for designing artificial reverberators," in Proc. 90th Convention Audio Engineering Society, Paris, France, preprint no. 3030, February, 1991.

[42] M. Karjalainen, A. Härmä, and U. K. Laine, "Realizable warped IIR filters and their properties," in Proc. IEEE International Conference on Acoustics, Speech, and Signal Processing, vol. 3, pp. 2205-2208, Munich, Germany, April 1997.

[43] A. Härmä, "Implementation of recursive filters having delay free loops," in Proc. IEEE International Conference on Acoustics, Speech, and Signal Processing, vol. 3, pp. 1261-1264, Seattle, Wash, USA, May 1998.

[44] P. W. Broome, "Discrete orthonormal sequences," Journal of the ACM, vol. 12, no. 2, pp. 151-168, 1965.

[45] A. V. Oppenheim, D. H. Johnson, and K. Steiglitz, "Computation of spectra with unequal resolution using the fast Fourier transform," Proceedings of the IEEE, vol. 59, pp. 299-301, 1971.

[46] G. Evangelista and S. Cavaliere, "Audio effects based on biorthogonal time-varying frequency warping," EURASIP Journal on Applied Signal Processing, vol. 2001, no. 1, pp. 2735, 2001.

[47] G. Evangelista and S. Cavaliere, "Auditory modeling via frequency warped wavelet transform," in Proc. European Signal Processing Conference, vol. I, pp. 117-120, Rhodes, Greece, September 1998.

[48] G. Evangelista and S. Cavaliere, "Dispersive and pitchsynchronous processing of sounds," in Proc. Digital Audio Effects Workshop, pp. 232-236, Barcelona, Spain, November 1998.

[49] G. Evangelista and S. Cavaliere, "Analysis and regularization of inharmonic sounds via pitch-synchronous frequency warped wavelets," in Proc. 1997 International Computer Music Conference, pp. 51-54, Thessaloniki, Greece, September 1997.

[50] L. Savioja and V. Välimäki, "Reducing the dispersion error in the digital waveguide mesh using interpolation and frequency-warping techniques," IEEE Trans. Speech and Audio Processing, vol. 8, no. 2, pp. 184-194, 2000.

[51] L. Savioja and V. Välimäki, "Multiwarping for enhancing the frequency accuracy of digital waveguide mesh simulations," IEEE Signal Processing Letters, vol. 8, no. 5, pp. 134-136, 2001.

[52] G. Evangelista, Dyadic Warped Wavelets, vol. 117 of Advances in Imaging and Electron Physics, Academic Press, NY, USA, 2001 . 
I. Testa was born in Napoli, Italy, on September 21, 1973. He received the Laurea in Physics from University of Napoli "Federico II" in 1997 with a dissertation on physical modeling of vibrating strings. In the following years, he has been engaged in the didactics of physics research, in the field of secondary school teacher training on the use of computer-based activities and in teaching computer architecture for the

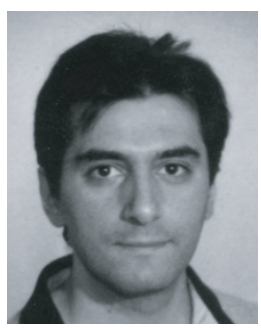
information sciences course. He is currently teaching "electronics and telecommunications" at the Vocational School, Galileo Ferraris, Napoli.

G. Evangelista received the Laurea in physics (with the highest honors) from the University of Napoli, Napoli, Italy, in 1984 and the M.S. and Ph.D. degrees in electrical engineering from the University of California, Irvine, in 1987 and 1990, respectively. Since 1995, he has been an Assistant Professor with the Department of Physical Sciences, University of Napoli "Federico II". From 1998 to 2002, he was a Scientific Ad-

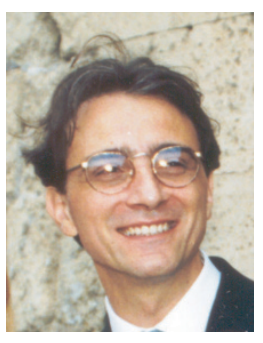
junct with the Laboratory for Audiovisual Communications, Swiss Federal Institute of Technology, Lausanne, Switzerland. From 1985 to 1986 , he worked at the Centre d'Etudes de Mathématique et Acoustique Musicale (CEMAMu/CNET), Paris, France, where he contributed to the development of a DSP-based sound synthesis system, and from 1991 to 1994, he was a Research Engineer at the Microgravity Advanced Research and Support Center, Napoli, where he was engaged in research in image processing applied to fluid motion analysis and material science. His interests include digital audio, speech, music, and image processing; coding; wavelets and multirate signal processing. Dr. Evangelista was a recipient of the Fulbright Fellowship.

S. Cavaliere received the Laurea in electronic engineering (with the highest honers) from the University of Napoli "Federico II", Napoli, Italy, in 1971. Since 1974, he has been with the Department of Physical Sciences, University of Napoli, first as a Research Associate and then as an Associate Professor. From 1972 to 1973, he was with CNR at the University of Siena. In 1986, he spent an academic year at the Media Lab-

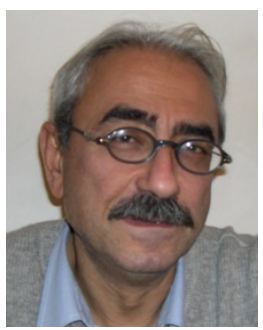
oratory, Massachusetts Institute of Technology, Cambridge. From 1987 to 1991, he received a research grant for a project devoted to the design of VLSI chips for real-time sound processing and for the realization of the Musical Audio Research Station, workstation for sound manipulation, IRIS, Rome, Italy. He has also been a Research Associate with INFN for the realization of very-large systems for data acquisition from nuclear physics experiments (KLOE in Frascati and ARGO in Tibet) and for the development of techniques for the detection of signals in high-level noise in the Virgo experiment. His interests include sound and music signal processing, in particular for the Web, signal transforms and representations, VLSI, and specialized computers for sound manipulation. 